\title{
Braking Force Model on Virus Transmission to Evaluate Interventions Including the Administration of COVID-19 Vaccines - Worldwide, 2019-2021
}

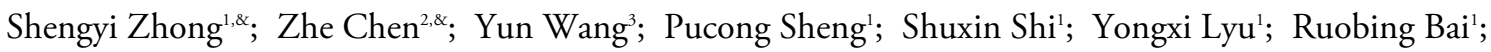 \\ Pengyu Wang'; Jiangjing Dong'; Jianbo $\mathrm{Ba}^{4}$; Xinmiao $\mathrm{Qu}^{5}$; Jian $\mathrm{Lu}^{6,7, * *}$
}

\begin{abstract}
Introduction: Assessing the effects of nonpharmaceutical interventions (NPIs) and vaccines on controlling the coronavirus disease 2019 (COVID-19) is key for each government to optimize the anticontagion policy according to their situation.

Methods: We proposed the Braking Force Model on Virus Transmission to evaluate the validity and efficiency of NPIs and vaccines. This model classified the NPIs and the administration of vaccines at different effectiveness levels and forecasted the duration required to control the pandemic, providing an indication of the future trends of the pandemic wave.

Results: This model was applied to study the effectiveness of the most commonly used NPIs according to the historic pandemic waves in different countries and regions. It was found that when facing an outbreak, only strict lockdown would give efficient control of the pandemic; the other NPIs were insufficient to promptly and effectively reduce virus transmission. Meanwhile, our results showed that NPIs would likely only slow down the pandemic's progression and maintain a low transmission level but fail to eradicate the disease. Only vaccination would likely have had a better chance of success in ending the pandemic.

Discussion: Based on the Braking Force Model, a pandemic control strategy framework has been devised for policymakers to determine the commencement and duration of appropriate interventions, with the aim of obtaining a balance between public health risk management and economic recovery.
\end{abstract}

\section{INTRODUCTION}

Since the outbreak of coronavirus disease 2019 (COVID-19), caused by severe acute respiratory syndrome coronavirus 2 (SARS-CoV-2) in December
2019 , the total number of related deaths worldwide has exceeded 2.8 million (1). Every country is currently taking various measures to combat the spread of this disease. However, eradication of this virus or stopping its transmission has been a challenging task. Evidence has shown herd immunity is unlikely to be achieved without intervention or vaccination (2-4). With the increase of daily new cases (5) and the lag in the production of antibodies by the vaccine and their uncertain effectiveness, it is urgent to first control the spread of COVID-19 via non-pharmaceutical interventions (NPIs). NPIs that limit social contact and enable continuance of protective behaviors, such as social distancing, night curfews, mask requirements, and area lockdowns, can help curb the pandemic until vaccines are rolled out (6-9).

Several countries have experienced three or more waves of the pandemic thus far. In most cases, outbreaks have occurred after the government loosened or withdrew the use of NPIs when the number of infections was lower than a certain threshold. To achieve an optimal balance between public health risks and economic recovery, in addition to the effects of the different NPIs, policymakers must understand the type of NPIs, i.e., mandatory or voluntary compliance, that should be implemented and during which phase of the pandemic these NPIs should be withdrawn.

While vaccines are being rolled out in several countries, it is still crucial to understand the types and timings of the different NPI measures that should be implemented to contain the pandemic effectively. Since the first COVID-19 vaccine was administered in the United Kingdom (UK) in December 2020, followed by numerous countries including the United States (US), Canada, some European countries, and China (10), studies on the effect of vaccines at each stage of the shifting population-vaccination ratio are being conducted. Most countries are still far from the goal of having more than $80 \%$ of their total population vaccinated. Implementing the most effective and 
appropriate NPIs during this transition period will help get people's life back to as close to normal as possible.

\section{METHODS}

\section{Braking Force Model}

A number of epidemiological transmission models have been established to evaluate and predict the increase in the number of COVID-19 cases (11-18). Some predictions were made based on the mobility of the virus spread represented by the basic reproduction number, $\mathrm{R}_{0}$. However, the parameters used in these models were complicated for two reasons. First, there are several unknown aspects of COVID-19, and it remains unclear as to why the virus mutates rapidly. Second, different social and environmental factors, such as government policies, environmental temperature, and population density, had different effects on these parameters. For instance, it would be inappropriate to use the epidemiological parameters of a cold, low-population-density country with good sanitary conditions to forecast the pandemic trajectory in a hotter, high population-density country with minimal government intervention measures.

This study developed a new model, called the Braking Force Model on Virus Transmission to examine and evaluate the validity and efficiency of different anti-contagion policies, including NPIs and vaccines under different situations and conditions and with different sample numbers. Most importantly, the Braking Force Model is not correlated to epidemiological parameters and it extracts information directly from the pandemic data. If we consider the pandemic to be a moving car, SARS-CoV-2 with its high transmissibility can be regarded as stepping on the accelerator, where the speed of spread or transmission of the virus is represented as $\mathrm{R}_{0}$. The higher the speed, the faster and wider is the spread of the pandemic. Governments brake the car by implementing different NPIs. In other words, whenever governments release the brakes, the car will pick up the speed again. Another way to slow down the car would be to increase the friction between the ground and the wheels of the car by making the ground extremely muddy or bumpy, such that the car stops - in other words, the pandemic stops, which is the desired effect of vaccination and can be achieved by breaking the chain of transmission. This analogy demonstrates that the speed of virus transmission is directly related to the dynamics of virus transmissibility, use of NPIs, and vaccination. These are the key factors determining the shape of the pandemic wave.

The proposed model can be expressed as follows:

$$
a_{\text {Covid } 19}=\mu_{\text {vaccine }} \cdot a_{R 0}+\sum a_{N P I} \cdot \gamma
$$

$a_{\text {Covidl } 9}=$ the acceleration of the pandemic at a particular point in time.

$a_{R 0}=$ acceleration of the basic reproduction number.

$\mathrm{R}_{0}=\mathrm{a}$ variable that depends on the mutation of the virus, temperature, and local population density.

$\mu_{\text {vacine }}=$ the coefficient or the vaccination ratio, which is 1 for no antibodies produced by vaccination and 0 when herd immunity is achieved.

$a_{N P I}=$ the acceleration of NPIs. This value is generally negative if it acts as the braking force. The deceleration, which is the absolute value of NPIs from high to low, is in the order of levels A, B, and C. Such a deceleration of the same NPI could vary according to the differences in factors such as anti-contagion policies, local sanitation, and the habits and customs of local people.

$\gamma=$ coefficient of execution efficiency of the NPIs; a multiplier of $a_{N P I}$.

To bring down the number of new cases, the absolute value of NPIs and vaccine deceleration must be higher than the basic acceleration of COVID-19. By studying the acceleration $a_{\text {Covidl } 9}$, we can assess the effect of each intervention by profiling the pandemic peak.

At present, policymakers need a model that can be easily adopted to analyze unknown epidemic transmission behaviors by identifying and foreseeing the growth of the pandemic based on the actual circumstances. Drawing from the widely used peak profile method in the field of physics, the Braking Force Model fits the wave without assuming any epidemiological parameters. It is expected that policymakers will be able to refer this model to examine the validity and efficiency of different anticontagion policies, including the use of NPIs and vaccines to achieve desirable and effective outcomes.

\section{Classification and Forecast}

Every increase and subsequent drop in new COVID-19 cases is described as a peak, or a wave. In our study, we extracted information using the peak profile method and determined the pandemic trajectory based on data from the database of new COVID-19 cases in different countries and regions (10). We first classified the epidemic control effectiveness manually into three levels. Level A efficiency represented a very efficient control of the 
pandemic, and the shape of the wave was fairly symmetric. Level B represented a mild control, with a longer tail in the wave shape. Level $\mathrm{C}$ represented an unsuccessful control with the number of daily new cases decreasing very slowly with repeated fluctuations (Supplymentary Figure S1, available in http://weekly. chinacdc.cn/).

Our data outlined the interval of the fitting parameters of each level. By using semi-supervised learning, our model can classify the ongoing wave and study the effect of different NPIs as well as vaccines in controlling the pandemic. Using the parameters obtained from peak fitting, our model can also forecast the pandemic tendencies of each ongoing wave under the current anti-contagion policy and provide a prediction parameter $\mathrm{t}_{30 \%}$ for each wave. This prediction parameter, $\mathrm{t}_{30 \%}$, represents the time required for the number of new cases to decrease to $30 \%$ of the highest number of new cases in a particular wave (see Supplementary Materials "forecast method" for detailed information, available in http://weekly. chinacdc.cn/), which could partially represent the speed of controlling the epidemic. A flow chart of the algorithm is presented in Figure 1. (Detailed information of the model and algorithm in the Supplementary Materials).

Compared to the classic epidemic model, one of the

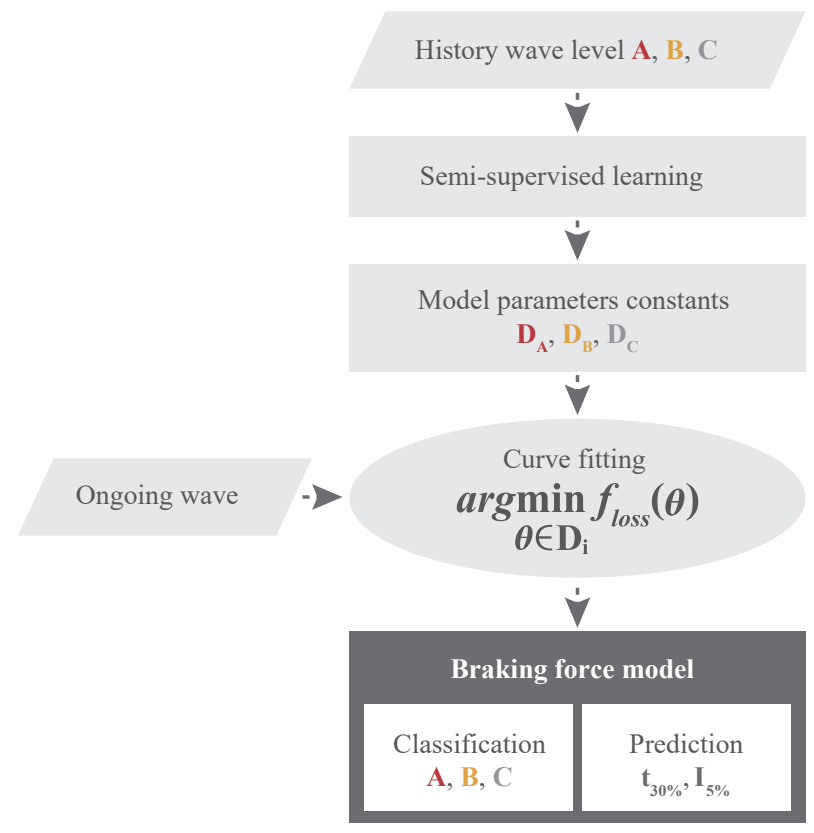

FIGURE 1. Flow chart of the classification and forecast of pandemic waves using the peak profile method.

Note: $1 \%$ : A parameter to predict the number of new daily cases as a percentage relative to the highest number of daily cases during the current wave of the pandemic. significant characteristics of the Braking Force Model lies in the fact that all the information is drawn directly from the pandemic data, i.e., historical daily new cases. No hypotheses are made on epidemiological transmissions, like the basic reproduction number $\left(\mathrm{R}_{0}\right)$, infection rate, or recovery rate, offering novel perspectives to understanding COVID-19.

\section{RESULTS}

\section{Braking Force Effects of NPIs}

We used the Braking Force Model to further study the effectiveness of the most commonly used NPIs, such as lockdowns, mask-wearing, and social distancing, on controlling the pandemic as well as their optimal implementation periods. Notably, the effectiveness of each NPI can vary according to people's actual behaviors in different countries and during different time periods. The classification is based on the assumption that the interventions are correctly and strictly implemented. Another assumption is that, in most parts of the world, the COVID-19 pandemic was much more severe in the second half of 2020. Hence, we study mainly the history of waves after July $2020(1,19)$, in order to achieve better comparisons between parameters.

\section{Level A Effectiveness - Lockdown}

As one of the most severe NPIs, lockdown is usually complemented with other NPIs such as social distancing, night curfews, and mask-wearing. We studied the data of five countries (Supplymenatary Table S1, available in http://weekly.chinacdc.cn/) and classified the effectiveness of lockdown on controlling COVID-19 as Level A, with an average prediction of $\mathrm{t}_{30 \%}$ of approximately 23 days. Its high effectiveness showed that lockdown is the fastest way to control the outbreak. However, lockdown causes serious societal and economic disruptions, which highlights the importance of finding the optimal duration. Thus, a prediction parameter $I_{5 \%}$ was set up for the daily number of new cases being $5 \%$ of the highest number of this wave. It is recommended that the lockdown policy should continue until it meets this 5\% parameter, i.e., when the current pandemic wave is under control.

\section{Lockdown Example: France}

The second round of lockdown in France occurred from October 30 to November 30 of 2020. After two weeks of lockdown, by incorporating the number of 
new infections on November 10, our Braking Force Model classified this wave as Level A with high efficiency and forecasted that the number of new infections could be reduced to $\mathrm{I}_{5 \%}$ on December 12 . In other words, our model suggested that by December 12, the wave of COVID-19 in France would be under control. It was found that such a forecast had close alignment with reality until the lockdown was lifted on November 30, when the number of new cases were approximately $25 \%$ of the peak. Subsequently, the daily new cases bounced back immediately and deviated from our forecast (Figure 2B).

\section{Lockdown Example: Belgium}

Belgium had a pandemic wave tendency and anticontagion policy similar to that of France before the end of November 2020. Belgium had also lifted their lockdown on December 1 at around the same time as France. However, at that time, the number of new cases in Belgium was approximately $\mathrm{I}_{15 \%}$; the mobility data (Supplymenatary Figure S3, available in http:// weekly.chinacdc.cn/) showed that Belgium succeeded to maintain a lower mobility trend than France and the number of new cases continued to decrease to $\mathrm{I}_{10 \%}$ around the time of Christmas. Despite the gap between reality and our projection of $\mathrm{I}_{5 \%}$, the pandemic stabilized eventually, as was predicted by our model (Figure 2B).

\section{Level B Effectiveness - Mask-Wearing}

If strictly and correctly implemented, mask-wearing can help reduce virus transmission with a high effectiveness of Level B, and sometimes, even Level A. The $t_{30 \%}$ of mask-wearing is commonly greater than the lockdown of Level A and the shape of wave is less symmetrical. However, its effectiveness is dependent on public behavior (20) and can drop to Level C if people do not strictly abide by the rules. For example, Singapore adopted a strict mask-wearing policy that required $95 \%$ of the population to wear a mask outside their homes, starting from September 2020 (20). According to our model, such policies have the potential to achieve Level B effectiveness, with an average $\mathrm{t}_{30 \%}$ for approximately 30 days (Supplementary Table S1).

\section{Level C Effectiveness - Night Curfews and Social Distancing}

The effectiveness of less strict NPIs, such as social distancing and night curfew were studied (Supplementary Table S1) based on the data of 10 countries and regions, including Germany, Sweden, and New York State. The effectiveness of social distancing was not found to be highly satisfactory, even when accompanied by the night curfew policy, mainly fluctuating between Level B to C, with an average $t_{30 \%}$ of approximately 44 and 48 days, respectively. By adopting these measures, a community will take at least twice as long to end the pandemic wave using less strict NPIs instead of lockdown.

By analyzing the history of the pandemic waves and the actual public response to the NPIs, we confirmed that leniency in implementing any anti-contagion policy would likely cause delays in pandemic control. Meanwhile rigorous execution of NPIs increases the braking force effectiveness. Consequently, when facing an outbreak, Level A measures should be implemented to promptly put the virus spread in control. Level B measures are also helpful but require additional time to curb the pandemic, whereas Level C NPIs have very limited contribution in this regard. Nevertheless, both Level B and C policies can be helpful for maintaining a low transmission level when the number of new cases decreases below $\mathrm{I}_{5 \%}$.

\section{Braking Force Effect of Vaccines}

With regard to investigating the effectiveness of vaccines in controlling the pandemic, our study focused on two countries, Israel and the United Arab Emirates (UAE), which had the highest vaccination ratios. We found that both these countries had inflection points of deceleration that were probably uncorrelated with the NPIs. The inflection point of the deceleration indicates the point at which the growth rate of epidemic cases has decreased, which in our model, is the date when the daily new cases start to decrease for a long period in the future. The inflection points of Israel took place on February 5, 2021, on which the braking force effect classification of its NPIs increased from Level $\mathrm{C}$ to Level $\mathrm{B}$. For the UAE, despite its continuous use of Level C NPIs, an inflection point was still observed on February 24 (Figure 3).

Since antibodies develop approximately 10 to 14 days after the vaccination, we found that the day when the vaccine was given was also when both Israel and the UAE reached 50 vaccine doses administered (VDA) per 100 people in the total population. While Israel had NPIs such as lockdown, few NPIs were implemented in the UAE, such as partial border closing. Since the inflection point of Israel occurred after the lockdown was called off, and the NPIs of the UAE remained stable before and after the inflection 
(A)

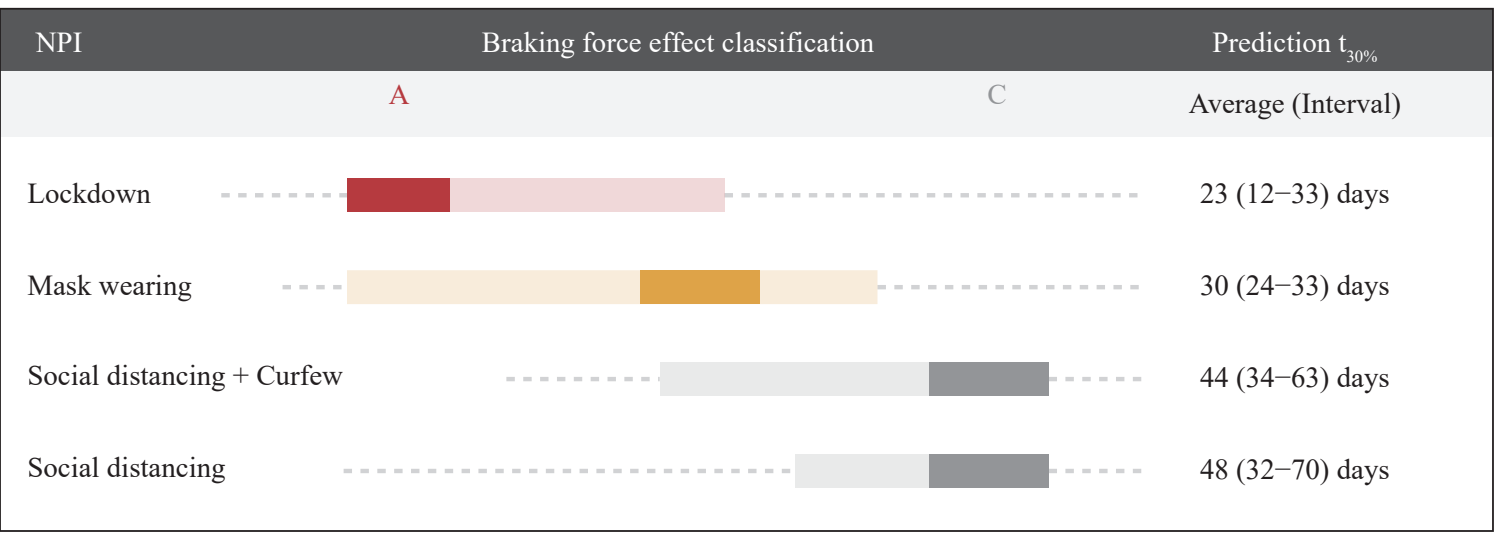
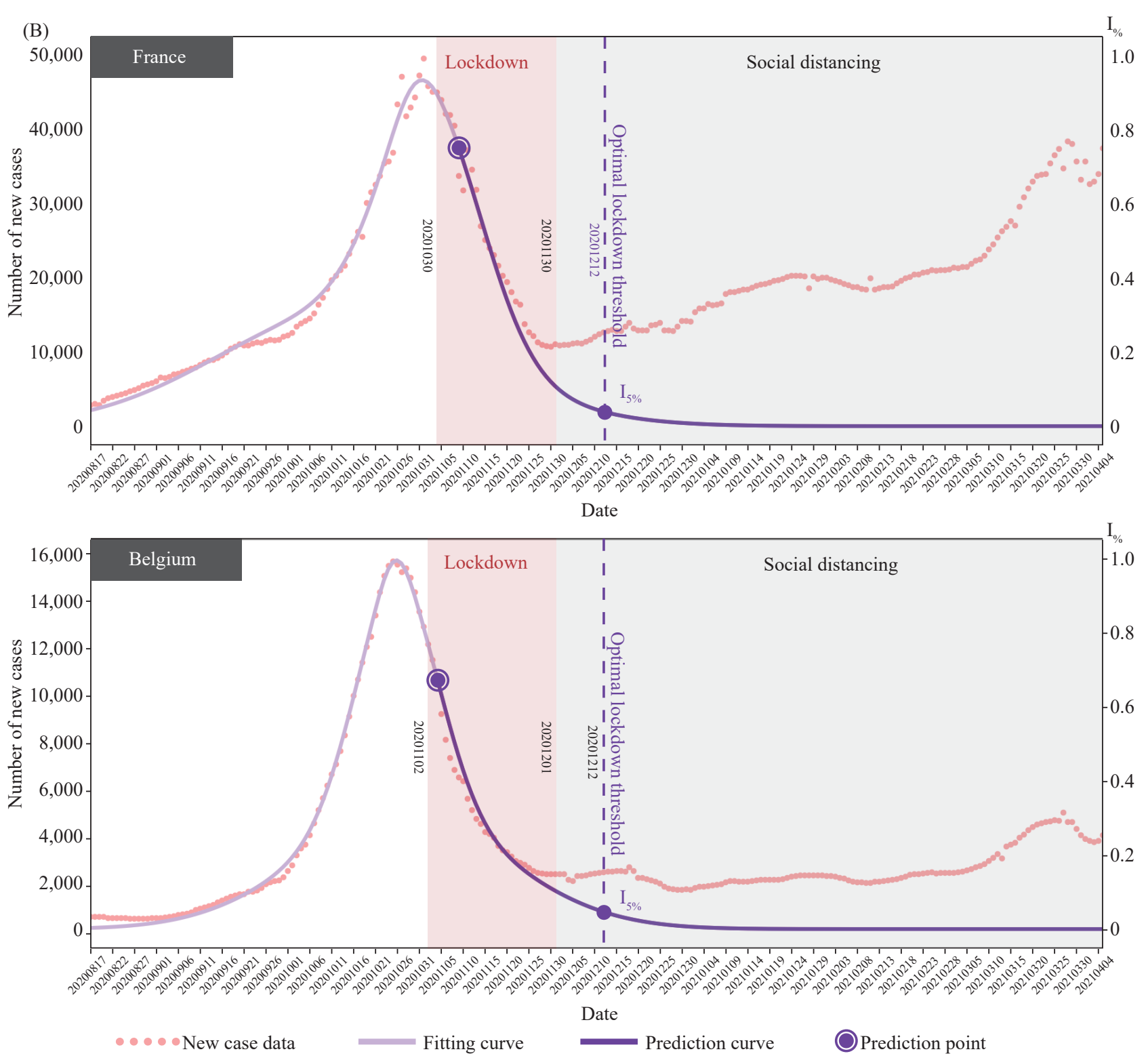

FIGURE 2. Non-pharmaceutical interventions (NPIs) classification and pandemic wave analysis. (A) Braking force effect classification of NPIs and their prediction $\mathrm{t}_{30 \%}$ value; $(B)$ analysis of pandemic wave of France and Belgium.

Note: $1 \%$ : A parameter to predict the number of new daily cases as a percentage relative to the highest number of daily cases during the current wave of the pandemic. 


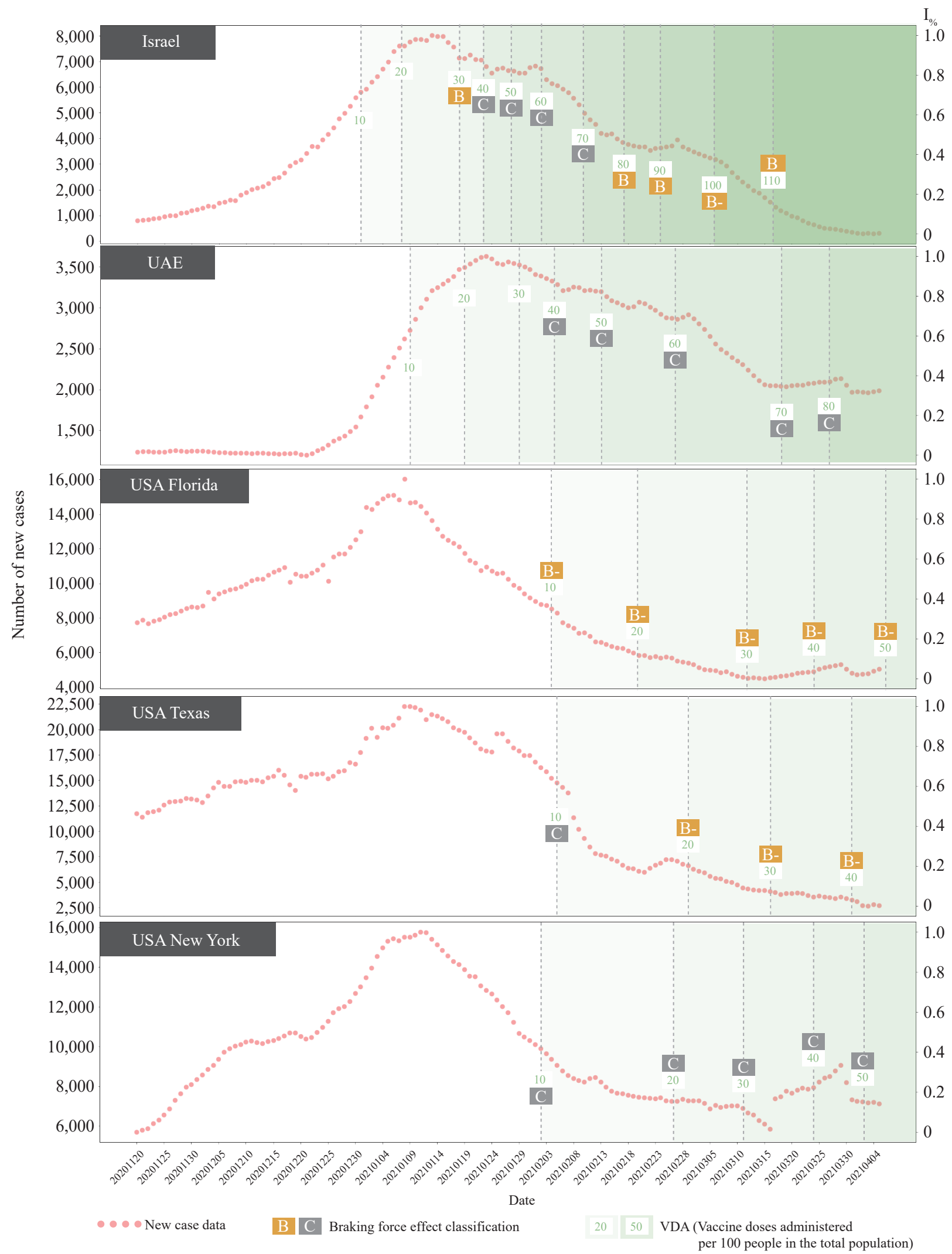

FIGURE 3. Braking Force Effectiveness classification of Israel, United Arab Emirates (UAE), and the United States (US) states of Florida, Texas, and New York with comparisons of different vaccination ratios.

Note: $1 \%$ : A parameter to predict the number of new daily cases as a percentage relative to the highest number of daily cases during the current wave of the pandemic. 
point, it was likely that the effectiveness of vaccination led to the inflection, so we could conclude that it is probably the vaccination played an important role in driving the wave to its inflection point.

In addition, Israel and UAE used different vaccines (Pfizer and Moderna for Israel, mRNA type and Sinopharm for UAE, inactivated type). These vaccines required two doses. Since the inflection point appeared synchronically around VDA $=50$, we could deduce that mRNA and inactivated vaccines showed an effect after $\mathrm{VDA}=50$. Before that, the contribution of the vaccine to controlling the pandemic was uncertain.

After incorporating the daily new cases in Israel on February 27, 2021, our model projected that the virus spread could be controlled to reach $\mathrm{I}_{5 \%}$ on April 3; this finding showed good alignment with the later trajectory (Supplementary Figure S4, available in http://weekly.chinacdc.cn/). For the UAE, if the current anti-contagion policy continues, $\mathrm{I}_{5 \%}$ should be reached around June 5, 2021 (Supplementary Figure S4). Theoretically, continuous vaccination will result in herd immunization. Since different manufacturers stated that their current vaccines would provide at least six months of immunity, it is crucial to achieve herd immunity in 6 months.

When evaluating vaccine effectiveness, the US was also studied because this country had both a large number of new cases as well as vaccine doses that were administered. We focused on three states in the US: Florida, New York, and Texas, wherein different NPIs were implemented. To date, their VDA had not reached the delay of $50+14$ days. Further, evident inflection points were observed. Notably, under similar VDA rates, the pandemic wave tendency and the braking force effectiveness classification of the three states were found to differ; thus, we can infer that the NPIs are the primary factors influencing the pandemic wave in the US.

\section{DISCUSSION}

Utilizing the Braking Force Model for COVID-19, we revealed the braking effect of NPIs and vaccines on the pandemic and provided a forecast method on when the pandemic could be controlled. Furthermore, this model also helps to propose a pandemic control strategy framework (Figure 4) when an outbreak occurs by implementing strict Level A NPIs, such as lockdown, to promptly and effectively curb virus transmission. In the first stage, the faster the spread of the pandemic can be restrained, the fewer the people who will be infected. Notably, the optimal threshold moment occurred when the number of new infections reduced to $5 \%$ of the summit of the wave, denoted $\mathrm{I}_{5 \%}$ (as predicted using our model); this was when the lockdown restrictions should be relaxed. Afterwards, less strict NPIs can be implemented to maintain the stability of the situation when the number of new cases is relatively low. In the second stage, there was a risk that the NPIs may become too lenient or relaxed, and

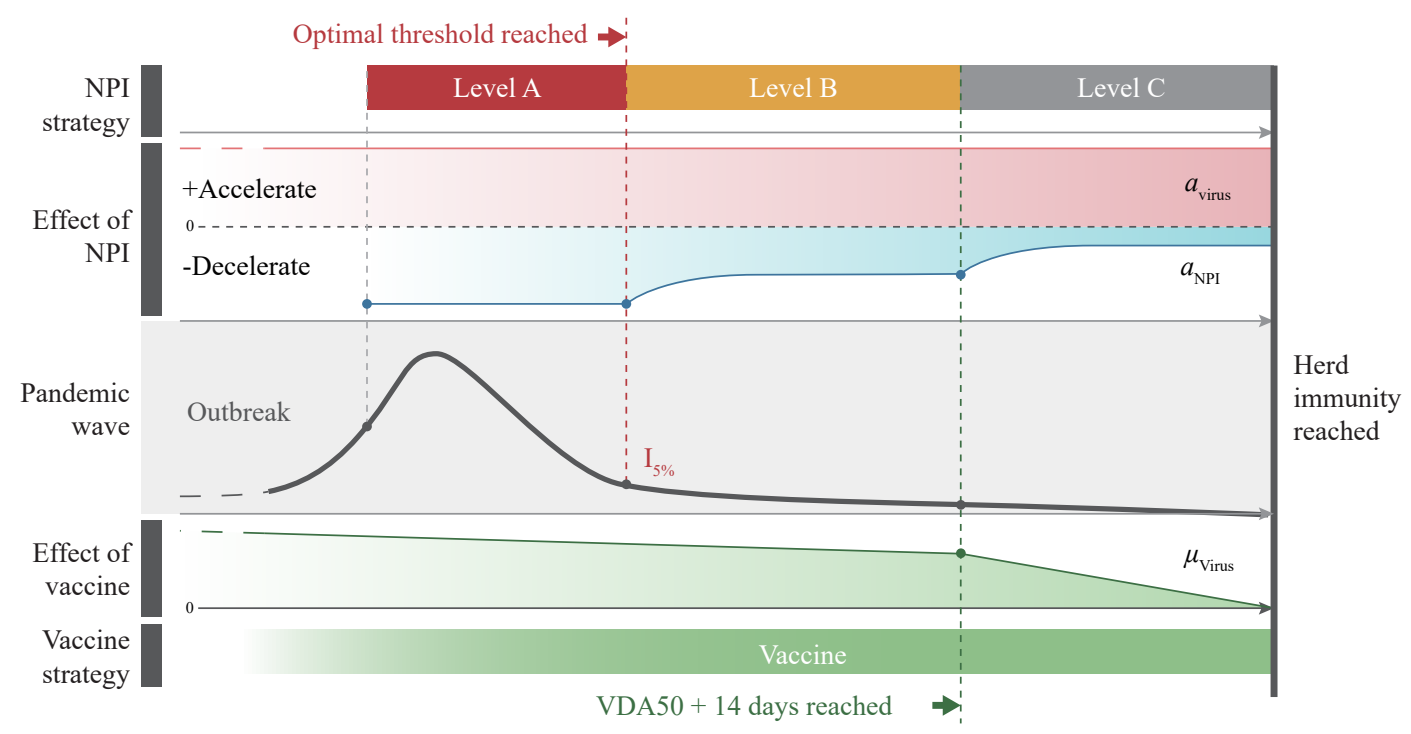

FIGURE 4. Anti-contagion strategy chart on COVID-19 obtained using the Braking Force Model.

Note: $1 \%$ : A parameter to predict the number of new daily cases as a percentage relative to the highest number of daily cases during the current wave of the pandemic.

Abbreviation: NPI=non-pharmaceutical interventions. 
this could lead to the sparking of a new outbreak.

To achieve herd immunity, vaccinating the majority of the population is paramount. Our study found that 14 days after $\mathrm{VDA}>50$, the vaccine had a remarkably decelerating effect on controlling the pandemic. In the last stage, NPIs can be made more flexible, allowing the resumption of social life to a certain extent.

According to our model, the UK is a good example that demonstrates the effectiveness of vaccination. In its previous wave from last December, a new mutant virus in addition to cold weather posed a very high risk for a serious outbreak in the UK. However, the UK government imposed a strict lockdown policy until April 1, when new infections reduced to roughly $\mathrm{I}_{5 \%}$. At the same time, they continued their NPI measures, such as social distancing and mask-wearing and administered a large number of vaccines. The VDA of the UK has reached 50 and the vaccination effect can be increased if the vaccination can be done within a short period of time, aiming to achieve herd immunity

Projection and recommendation of the anticontagion policy for several countries and regions by using the Braking Force Model can be found below (Supplementary Figures S5-S6, available in http:// weekly.chinacdc.cn/). The limitation of this model is that it provides less information during the pandemic. However, this model offers insights into the dynamics of NPIs and vaccines in the pandemic with the help of peak or wave shape data analysis, and the results are easier to understand than those provided by conventional epidemic models.

The Braking Force model presented a new paradigm to predict the evolution of the COVID-19 pandemic. The effectiveness of different NPIs and vaccination were analyzed to help policymakers perform better forecasts with the measures they chose to implement. Our results showed that NPI intervention could likely only slow down the pandemic evolution but failed to eradicate the disease. Only vaccination had a higher likelihood of ending the pandemic, starting with VDA with approximately $50+14$ days of delay for antibody production. The UK and Israel were close to the end of the national-level pandemic situation by successfully combining NPIs and high coverage of effective vaccinations.

Funding: Shenzhen-Hong Kong Science and Technology Innovation Cooperation Zone Shenzhen Park Project: HZQB-KCZYB-2020030, the National Natural Science Foundation of China No. 11875192; Chinese Academy of Sciences ANSO-SBA-2020-06; and Hong Kong Innovation and Technology
Commission via the Hong Kong Branch of National Precious Metals Material Engineering Research Centre.

doi: $10.46234 / \mathrm{ccdcw} 2021.195$

\# Corresponding author: Jian Lu, jianlu@cityu.edu.hk.

SJTU - Paristech Institute of Technology, Shanghai Jiao Tong University, Shanghai, China; ${ }^{2}$ School of materials science and engineering, Shanghai Jiao Tong University, Shanghai, China; ${ }^{3}$ School of Mechanical Engineering and Automation, Beihang University, Beijing, China; ${ }^{4}$ Naval Medical Centre, Naval Medical University, Shanghai, China; ${ }^{5}$ School of Data Science, City University of Hong Kong, Hong Kong, China; ${ }^{6}$ Department of Biomedical Science, City University of Hong Kong, Hong Kong, China; ${ }^{7}$ Hong Kong Branch of National Precious Metals Material Engineering Research Center (NPMM), City University of Hong Kong, Hong Kong, China.

$\&$ Joint first authors.

Submitted: August 16, 2021; Accepted: September 01, 2021

\section{REFERENCES}

1. World Health Organization. Coronavirus disease (COVID-19) weekly epidemiological update and weekly operational update. 2021. https: www.who.int/emergencies/diseases/novel-coronavirus-2019/situationreports/. [2021-4-10].

2. Saad-Roy CM, Wagner CE, Baker RE, Morris SE, Farrar J, Graham $\mathrm{AL}$, et al. Immune life history, vaccination, and the dynamics of SARSCoV-2 over the next 5 years. Science 2020;370(6518):811-8. http://dx.doi.org/10.1126/science.abd7343.

3. Cobey S. Modeling infectious disease dynamics. Science 2020;368 (6492):713 - 4. http://dx.doi.org/10.1126/science.abb5659.

4. Pei S, Kandula S, Shaman J. Differential effects of intervention timing on COVID-19 spread in the United States. Sci Adv 2020;6 (49):eabd6370. http://dx.doi.org/10.1126/SCIADV.ABD6370.

5. Davies NG, Abbott S, Barnard RC, Jarvis CI, Kucharski AJ, Munday JD, et al. Estimated transmissibility and impact of SARS-CoV-2 lineage B.1.1.7 in England. Science 2021;372(6538):eabg3055. http://dx. doi.org/10.1126/science.abg3055.

6. Lai SJ, Ruktanonchai NW, Zhou LC, Prosper O, Luo W, Floyd JR, et al. Effect of non-pharmaceutical interventions to contain COVID-19 in China. Nature 2020;585(7825):410 - 3. http://dx.doi.org/10.1038/ s41586-020-2293-x.

7. Flaxman S, Mishra S, Gandy A, Unwin HJT, Mellan TA, Coupland H, et al. Estimating the effects of non-pharmaceutical interventions on COVID-19 in Europe. Nature 2020;584(7820):257 - 61. http://dx. doi.org/10.1038/s41586-020-2405-7.

8. Soltesz K, Gustafsson F, Timpka T, Jaldén J, Jidling C, Heimerson A, et al. The effect of interventions on COVID-19. Nature 2020;588 (7839):E26 - 28. http://dx.doi.org/10.1038/s41586-020-3025-y.

9. Hsiang S, Allen D, Annan-Phan S, Bell K, Bolliger I, Chong T, et al. The effect of large-scale anti-contagion policies on the COVID-19 pandemic. Nature 2020;584(7820):262 - 7. http://dx.doi.org/10.1038/ s41586-020-2404-8.

10. Mathieu E, Ritchie H, Roser M. Our World in Data is now tracking Coronavirus (COVID-19) vaccinations across the world. 2021. https://ourworldindata.org/covid-vaccination-dataset. [2021-4-10].

11. Pollán M, Pérez-Gómez B, Pastor-Barriuso R, Oteo J, Hernán MA, Pérez-Olmeda M, et al. Prevalence of SARS-CoV-2 in Spain (ENECOVID): a nationwide, population-based seroepidemiological study. Lancet 2020;396(10250):535 - 44. http://dx.doi.org/10.1016/S01406736(20)31483-5.

12. Chang S, Pierson E, Koh PW, Gerardin J, Redbird B, Grusky D, et al. Mobility network models of COVID-19 explain inequities and inform reopening. Nature 2021;589(7840):82 - 7. http://dx.doi.org/10.1038/ s41586-020-2923-3. 
13. Kraemer MUG, Yang CH, Gutierrez B, Wu CH, Klein B, Pigott DM, et al. The effect of human mobility and control measures on the COVID-19 epidemic in China. Science 2020;368(6490):493-7. http://dx.doi.org/10.1126/science.abb4218.

14. Tian HY, Liu YH, Li YD, Wu CH, Chen B, Kraemer MUG, et al. An investigation of transmission control measures during the first 50 days of the COVID-19 epidemic in China. Science 2020;368(6491):638 42. http://dx.doi.org/10.1126/science.abb6105.

15. Walker PGT, Whittaker C, Watson OJ, Baguelin M, Winskill P, Hamlet A, et al. The impact of COVID-19 and strategies for mitigation and suppression in low- and middle-income countries. Science 2020;369(6502):413 - 22. http://dx.doi.org/10.1126/science.abc0035.

16. Britton T, Ball F, Trapman P. A mathematical model reveals the influence of population heterogeneity on herd immunity to SARS-CoV2. Science 2020;369(6505):846 - 9. http://dx.doi.org/10.1126/science. abc6810

17. Lu J. A new, simple projection model for COVID-19 pandemic. medRxiv 2020. http://dx.doi.org/10.1101/2020.03.21.20039867.

18. Sun KY, Wang W, Gao LD, Wang Y, Luo KW, Ren LS, et al. Transmission heterogeneities, kinetics, and controllability of SARSCoV-2. Science 2021;371(6526):eabe2424. http://dx.doi.org/10.1126/ science.abe2424.

19. Blacatnik School of Government University, University of Oxford. COVID-19 government response tracker. 2021. https://www.bsg. ox.ac.uk/research/research-projects/covid-19-government-responsetracker. [2021-4-10].

20. Imperial College London. COVID-19 behavior tracker. 2021. https:// www.imperial.ac.uk/centre-for-health-policy/our-work/our-response-tocovid-19/covid-19-behaviour-tracker/. [2021-4-10]. 


\section{Supplementary Materials}

The structure of Materials and Methods is as follows: we first introduce the construction process of our modelspecific database in the "Datasets" section. Then, in the "Model" section, the origin of final parameter interval for different classes is explained. Finally, in the "Classification and forecast method" section, the detailed procedure for classifying the observed curve of daily reported cases and predicting the development is presented.

\section{Storage of epidemic COVID-19 curve}

\section{Datasets}

From the dataset of World Health Organization (1) and Our World in Data (2), we collected and saved the daily new cases of COVID-19 covering the data from January 22, 2020 to April 9, 2021 from more than 60 countries and regions. The date is accurate to the day, however, due to different conditions, statistical methods and administrative implementation between countries, the data vary greatly in form and in scale. In our study, these data were presented in form of curves by setting date as horizontal axis and the number of daily new cases as vertical axis.

\section{Process of epidemic COVID-19 curve}

In order to facilitate the extraction and the analysis of features of the original data, a preliminary process was introduced. The pre-processing contained two steps. The first step was smoothing the data. The number of each day was smoothed by the data of seven days before and after, which means we replaced the number of that day by the average value of the seven days. This procedure can partly remove the noise in the data and help reflect the trend. Secondly, we divided the data of each country/region into a minor level: waves. Each wave represents a round of outbreak and mitigation of COVID-19. The following rule were used to identify a wave: it starts from the closest

SUPPLEMENTARY TABLE S1. Classification of different non-pharmaceutical interventions of history wave and their forecast parameter $t_{30 \%}$.

\begin{tabular}{|c|c|c|c|c|c|}
\hline Non-pharmaceutical interventions & Country/Region & Period & Classification & $\mathbf{t}_{30 \%}$ & Average $t_{30 \%}$ \\
\hline \multirow{5}{*}{ Lockdown } & France & $2020.8-2020.12$ & A & 23 & \multirow{5}{*}{23} \\
\hline & South Africa & 2020.9-2021.3 & $A$ & 12 & \\
\hline & Belgium & $2020.8-2020.12$ & $A$ & 14 & \\
\hline & Austria & $2020.8-2021.1$ & A- & 33 & \\
\hline & UK & 2020.9-2021.3 & $\mathrm{B}$ & 32 & \\
\hline \multirow{6}{*}{ Mask wearing } & The Republic of Korea * & $2020.2-2020.4$ & $A$ & 12 & \multirow{3}{*}{ l } \\
\hline & Hong Kong * & $2020.3-2020.5$ & A- & 16 & \\
\hline & Japan * & $2020.1-2020.3$ & B & 22 & \\
\hline & Japan & 2020.9-2021.1 & $\mathrm{B}$ & 24 & \multirow{3}{*}{30} \\
\hline & The Republic of Korea & 2020.10-2021.2 & $\mathrm{B}-$ & 33 & \\
\hline & Spain & 2020.7-2020.11 & $\mathrm{B}-$ & 32 & \\
\hline \multirow{5}{*}{ Social distancing + Curfew } & Texas & 2020.9-2021.3 & B & 34 & \multirow{5}{*}{44} \\
\hline & Hungary & $2020.8-2021.1$ & $\mathrm{~B}-$ & 44 & \\
\hline & Illinois & 2020.9-2021.1 & C & 42 & \\
\hline & Florida & 2020.9-2021.2 & $\mathrm{C}$ & 35 & \\
\hline & Slovenia & 2020.8-2021.3 & $\mathrm{C}$ & 63 & \\
\hline \multirow{5}{*}{ Social distancing } & lowa & 2020.9-2021.1 & B- & 32 & \multirow{5}{*}{48} \\
\hline & New York & 2020.9-2021.2 & $\mathrm{B}-$ & 57 & \\
\hline & Germany & 2020.8-2021.2 & B- & 44 & \\
\hline & Switzerland & $2020.8-2021.2$ & C & 35 & \\
\hline & Sweden & 2020.9-2021.2 & $\mathrm{C}$ & 70 & \\
\hline
\end{tabular}

* The history waves were not counted into the average value of $\mathrm{t}_{30 \%}$. 
inflection point before its augmentation and ends on the closest inflection point after its decline. The inflection point was defined as the points between two local maximums of the smoothed data around which the data remained stable. The following research will mainly focus on the waves of the smoothed data of all the sample countries.

\section{Classification of epidemic curve in training set}

We subdivided the original epidemic data into wave-based data and randomly divided them into a training set and a test set at a ratio of $8: 2$. The training set was used to build the model, and the test set was used to test the validity of the model. We manually classified the epidemic wave curve in the training set into three levels: $\mathrm{A}, \mathrm{B}$, and $\mathrm{C}$ based on the pandemic control efficiency in previous text, part "Classification and forecast of the braking force effect" with level A being the highest and level C being the lowest (Supplementary Figure S1). Therefore, the database contained the classified training set and the test set to be verified. The data in both sets were based on the smoothed epidemic curve data of each wave in each sample country/region.

\section{Initial parameter intervals}

\section{Model}

To obtain the initial parameter interval of each Braking Force Effect Level, epidemic curves in training set were processed. Since the Gaussian function was frequently used to describe the natural phenomenon including the spread of infectious disease, while the epidemic curve usually has strong fluctuation, our study applied a linear combination of three Gaussian functions to fit the epidemic curve, which could be expressed as follow:

$$
f(x)=a_{1} \frac{e^{-\left(x-\mu_{1}\right)^{2}}}{2 \sigma_{1}^{2}}+a_{2} \frac{e^{-\left(x-\mu_{2}\right)^{2}}}{2 \sigma_{2}^{2}}+a_{3} \frac{e^{-\left(x-\mu_{3}\right)^{2}}}{2 \sigma_{3}^{2}}
$$

In which, $a_{i}, \mu_{i}, \sigma_{i}$ are the amplitudes, mean value and standard deviation of each Gaussian function. Loss function is defined as the mean-square error $\delta(X)=\sum_{i=1}^{n} \frac{\left(y_{i}-f\left(x_{i}\right)\right)^{2}}{n}$ in which $n$ is number of points in the origin epidemic curve, $x_{i}$ and $y_{i}$ are the abscissa and ordinate of the corresponding point.

Since there were nine parameters to fit, an algorithm combining the Bayesian algorithm and gradient descent method was proposed to enhance the speed and accuracy, in which the Bayesian algorithm could quickly find a feasible solution close to the optimal solution in the search space and the gradient descent method was used to effectively approximate the optimal solution.

Through the above method, the epidemic wave in the training set could be quickly and accurately fitted by the linear combination of three Gaussian functions. After each fitting, it generated a set of data containing nine corresponding parameters, therefore the initial parameter intervals of each level were formed.

Final parameter intervals. Albeit the initial parameter had been found, the classification and forecast capability of the model was not satisfied. The reason was that the nine independent parameters intervals cannot effectively represent the three classes of epidemic curve. Therefore, feature parameters have been setup in order to reduce the
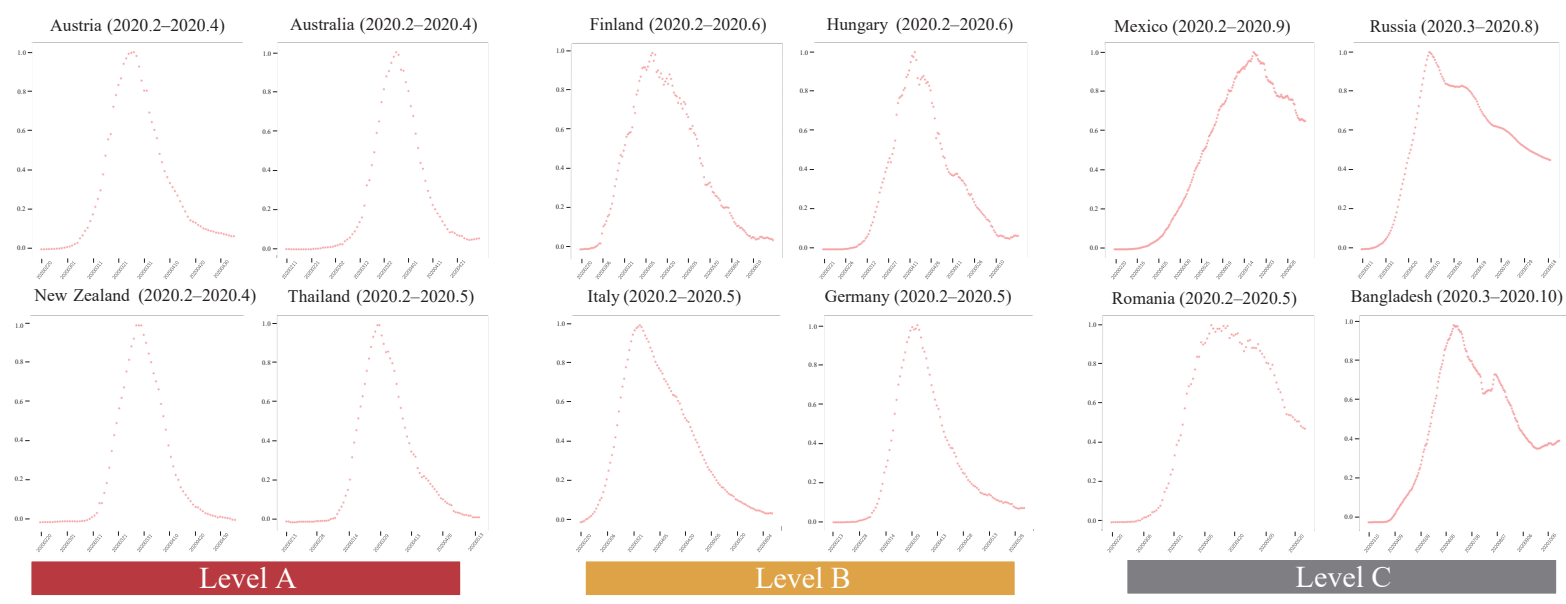

SUPPLEMENTARY FIGURE S1. Samples of pandemic wave classified into braking force effect level A, B, and C according to the pandemic control effectiveness. 


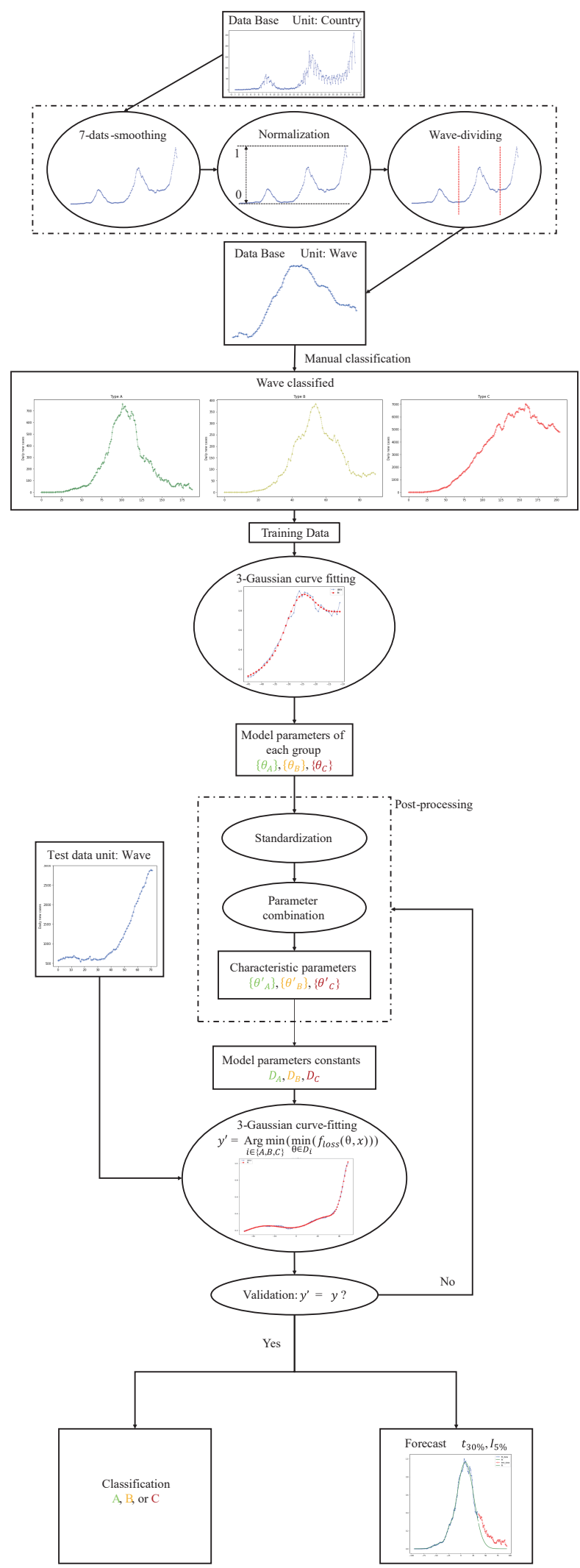

SUPPLEMENTARY FIGURE S2. Flow chart of classification and forecast with detail information of the Braking Force Effect model above the caption.

Note: $1 \%$ : A parameter to predict the number of new daily cases as a percentage relative to the highest number of daily cases during the current wave of the pandemic. 
Retail and recreation: How did the number of visitors change since the beginning of the pandemic?

This data shows how the number of visitors to places of retail and recreation has changed relative to the period before the pandemic.

This includes places like restaurants, cafes, shopping centers, theme parks, museums, libraries, movie theaters.

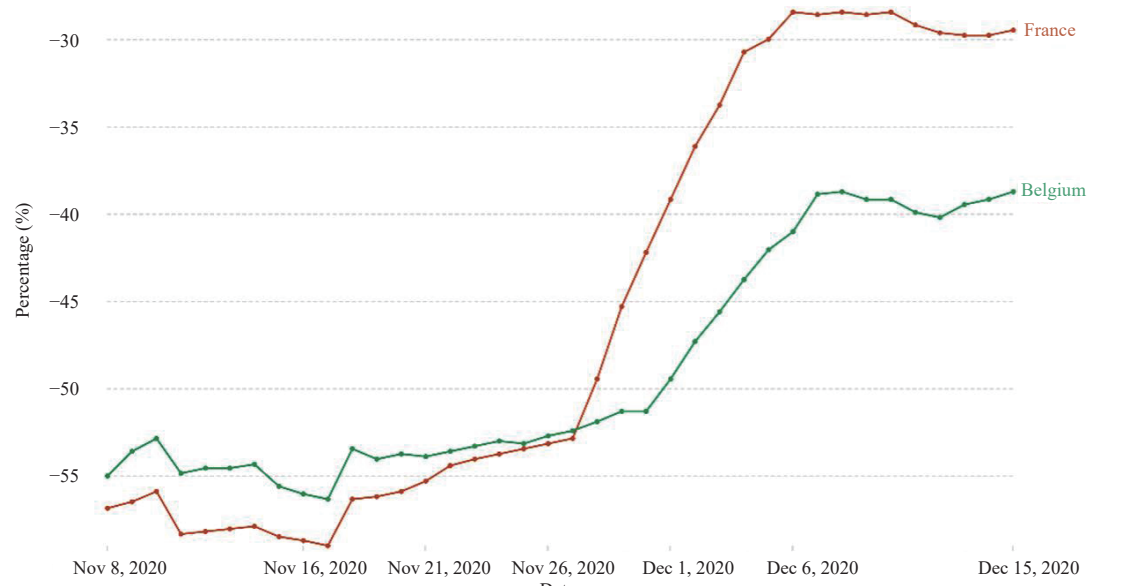

SUPPLEMENTARY FIGURE S3. Community mobility trends of France and Belgium during the period of November 8 to December 15, 2020 (1).
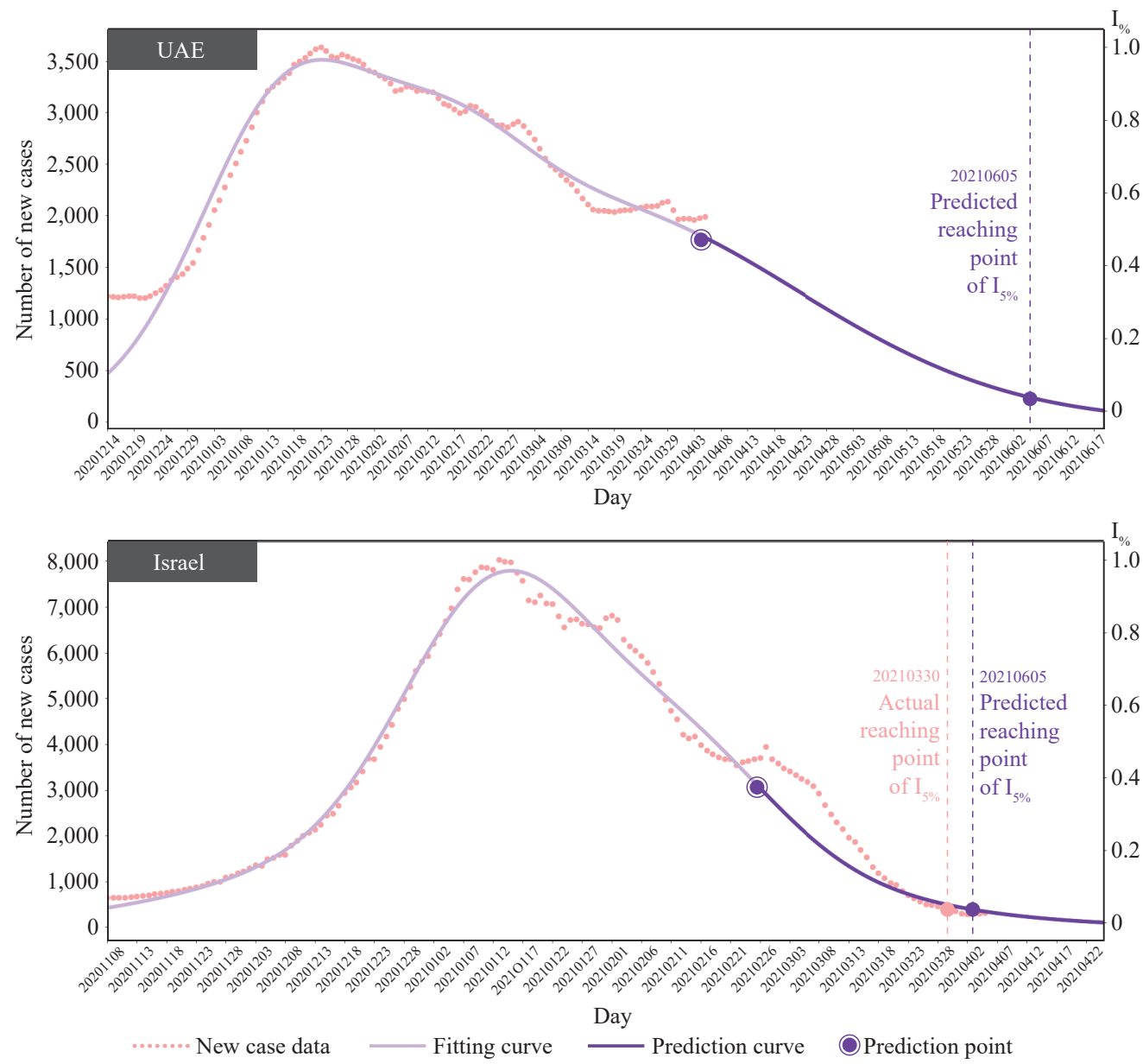

SUPPLEMENTARY FIGURE S4. Forecast of current pandemic wave of Israel and UAE.

Note: $1 \%$ : A parameter to predict the number of new daily cases as a percentage relative to the highest number of daily cases during the current wave of the pandemic. 

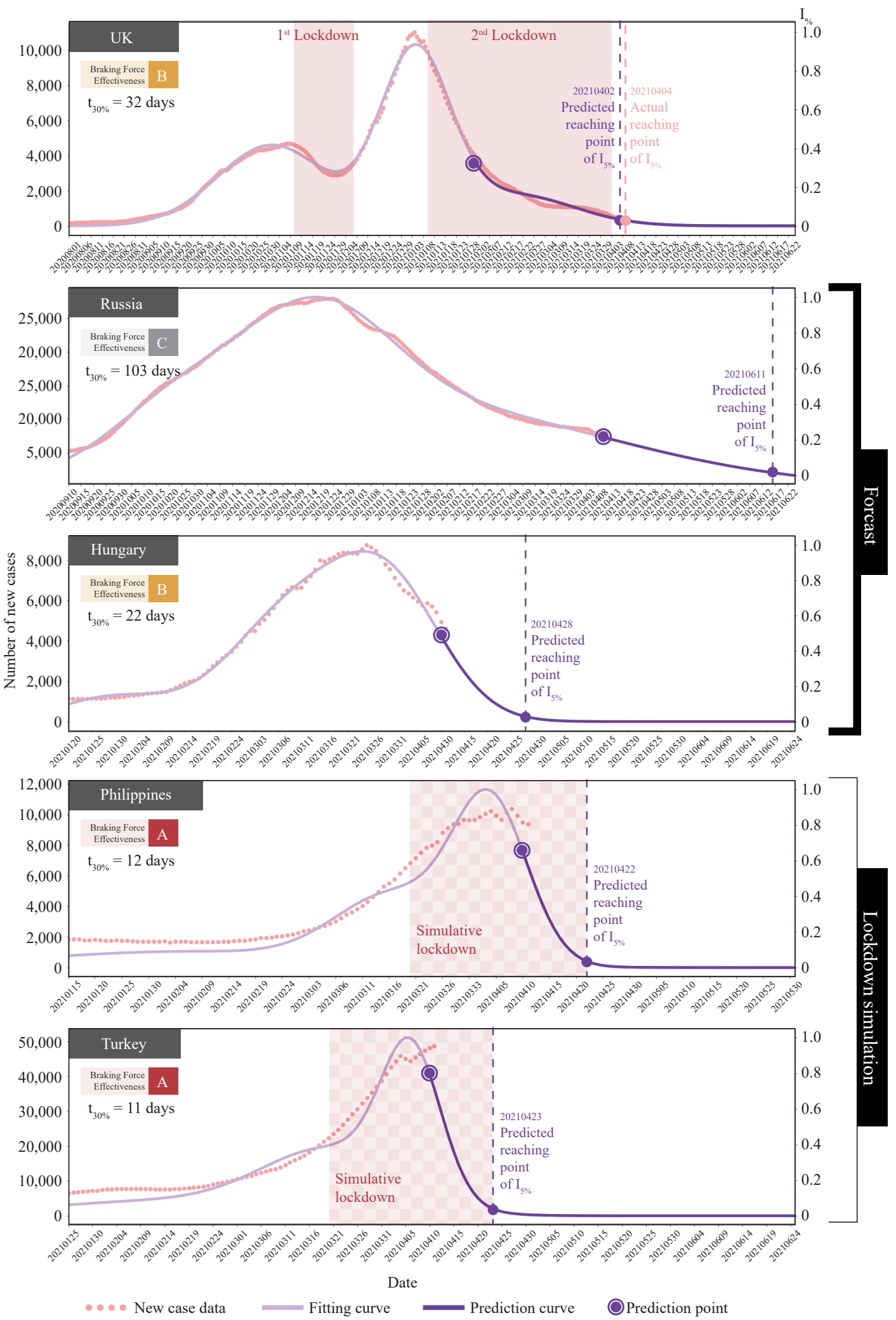

SUPPLEMENTARY FIGURE S5. "Braking Force Effect" model application. (A) "Braking Force Effect" model application to UK; (B) forecast of COVID-19 new case of Russia and Hungary. (C) "Braking force effect" model simulation of new case number tendency if The Philippines and Turkey implement Level A NPIs.

Note: 1\%: A parameter to predict the number of new daily cases as a percentage relative to the highest number of daily cases during the current wave of the pandemic. 


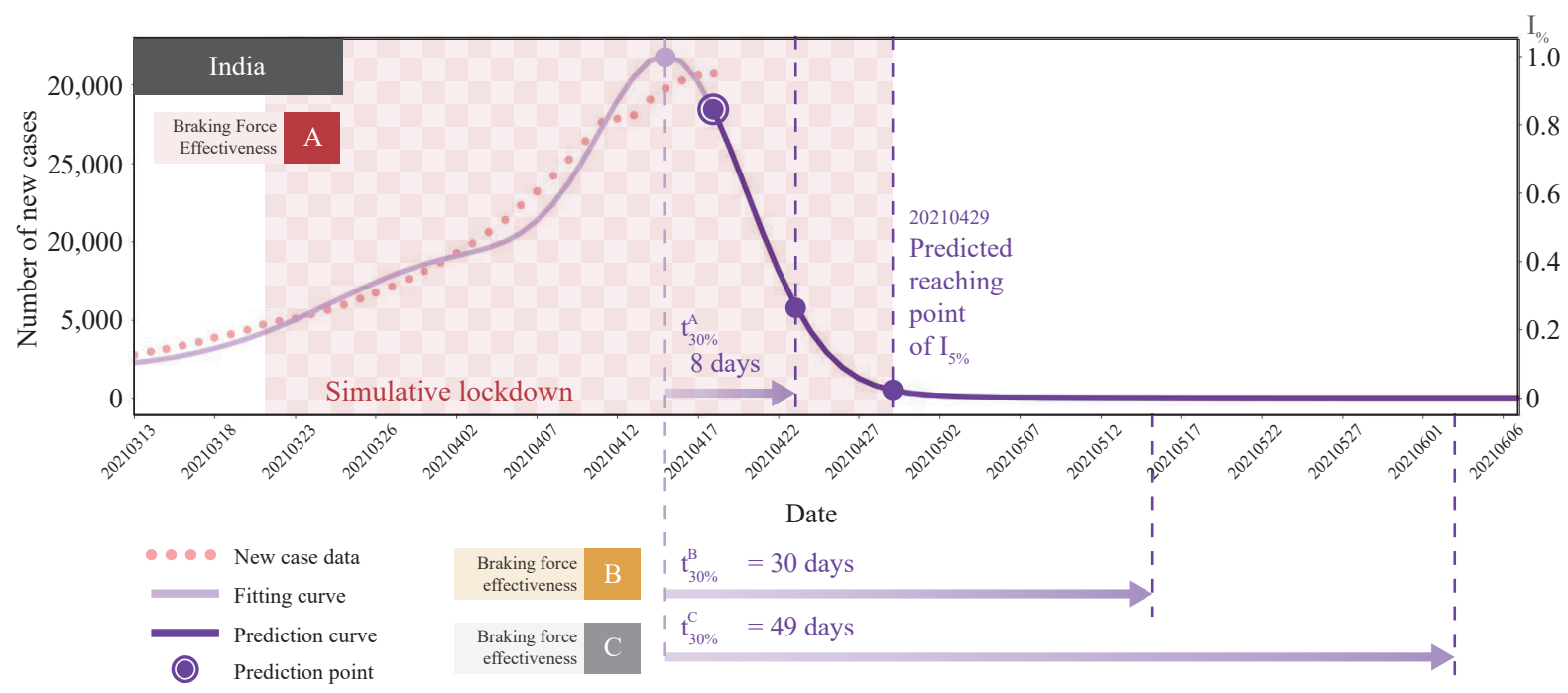

SUPPLEMENTARY FIGURE S6. "Braking Force Effect" model simulation of new case number tendency for India with different Braking Force Effectiveness.

Note: $1 \%$ : A parameter to predict the number of new daily cases as a percentage relative to the highest number of daily cases during the current wave of the pandemic.

initial parameter interval as well as to improve the classification and forecast capabilities of the model.

The feature parameters are $a_{1}, a_{2}, a_{3 r}, \mu_{1_{n}}, \mu_{2_{n}}, \mu_{3_{n}}, \sigma_{1_{n}}, \sigma_{2_{r}}, \sigma_{3_{r}}$, where $a_{i_{r}}=a_{i} / a_{1}, i=2,3 ; \mu_{i_{r}}=a_{i} / N, i=1,2,3$; $\sigma_{1_{n}}=\sigma_{1} / N ; \sigma_{i_{r}}=\sigma_{i} / \sigma_{1}, i=2,3$ and $N$ is the number of sampling points. The selection of index $i$ is to evaluate the influence of the second and third peak to the main peak to estimate the fluctuation of the epidemic curve. The selection of index $n$ is to limit the range of $\mu$ and $\sigma$ when processing a long-term epidemic wave. Through the limitation of these new feature parameters, the final parameter intervals were calculated, and it was found that their range is greatly limited comparing to the initial parameter intervals. As a result, the overlap between each class in the nine-dimension space was reduced, the capability of classification was effectively improved.

\section{Characteristic parameters}

Besides the feature parameters used to calculate the final parameter interval mentioned above, characteristic parameters have been setup to assist the classification. The boxplot was used to exploit these parameters in order to distinguish the interval of each level. Characteristic parameters that meet the above requirement could be expressed below which were mainly related to $a_{1}, a_{2}, a_{3}, \sigma_{2}$, because these parameters would greatly influence the fluctuation of epidemic curve.

$$
\begin{aligned}
& a_{s q r}=\frac{\sqrt{a_{2}^{2}+a_{3}^{2}}}{a_{1}}, \quad a_{3_{r}}=\frac{a_{3}}{a_{1}}, \quad a_{23_{r}}=\frac{a_{2} \times a_{3}}{a_{1}^{2}}, \\
& t_{2}=a_{23_{r}} \times a_{2_{r}} \times \sigma_{2_{r}}, \text { etc. }
\end{aligned}
$$

These characteristic parameters of epidemic wave in the training set of each level presented a certain interval, and the scatter chart between 2 characteristic parameters, e.g., $a_{s q r} V_{s} a_{3_{r}}$ or $a_{23_{r}} V_{s} t_{2}$, presented a characteristic spatial distribution and slope range. By analyzing the characteristic parameters and the scatter charts, we can classify the types of one epidemic wave.

\section{Classification method}

\section{Classification and Forecast Method}

To classify the existing curve of each wave according to the criteria mentioned in the "Datasets" section, our standard procedure was divided into three steps. First, fit respectively the existing wave within the final parameter intervals of three levels obtained in the "Model adjustment" part by using the linear combination of three Gaussian functions. Second, classify preliminarily the waves by choosing the level with the smallest chi-squared value, if the chi-squared value was almost equally to two neighbor level, e.g. "A and B" and "B and C," the wave would be 
classified as A- and B-, respectively. Third, calculate the corresponding characteristic parameters and their relative scatter charts. By comparing the relative distance to other points of three levels and the slope from the position to the origin, the auxiliary result could be obtained to judge quantitatively whether the preliminary classification is reasonable. If the result between preliminary and auxiliary classification was the same then the former judgment would be accepted; otherwise, the wave would be reclassified. By repeating the classification until they were agreed, the final classification could be obtained, including A, A-, B, B-, C.

\section{Forecast Method}

To predict the number of daily reported cases, the nine parameters of the linear combination of three Gaussian function were firstly calculated according to the procedure in "Classification Method" part. The wave thus could be extended as the forecast result. The flow chart of classification and forecast with detail information of the "braking force effect" model is presented in Supplementary Figure S3.

During the forecast, a variable named $\mathrm{t}_{30 \%}$ was defined for indicating the decrease velocity of pandemic wave, unit of days, presented as the number of days required to decrease from the summit of wave to its $30 \%$. We supposed a wave normalized as $w: \llbracket 0, N \rrbracket \rightarrow[0,1]$ and the $\mathrm{t}_{30 \%}$ defined as:

$$
t_{30 \%}=\min (\{n \in \llbracket 0, N \rrbracket, \forall i \in \llbracket n, N \rrbracket, w(i) \leq 0.3 \times \max (w)\})
$$

A wave with a smaller $t_{30 \%}$ value means the pandemic have been efficiently controlled. History waves of different countries/regions with different implemented NPIs were classified and listed in Supplementary Table S1. $t_{30 \%}$ of each history wave were calculated, and the average $\mathrm{t}_{30 \%}$ of each corresponding NPIs are presented in the table. Average value of $\mathrm{t}_{30 \%}$ were only for the history wave after July 2020, considering that in most parts of the world, the new case number of the COVID-19 after July 2020 was much higher than before.

\section{REFERENCES}

1. World Health Organization. Coronavirus disease (COVID-19) weekly epidemiological update and weekly operational update. 2021. https:www.who.int/emergencies/diseases/novel-coronavirus-2019/situation-reports/. [2021-4-10].

2. Mathieu E, Ritchie H, Roser M. Our World in Data is now tracking Coronavirus (COVID-19) vaccinations across the world. 2021. https:// ourworldindata.org/covid-vaccination-dataset. [2021-4-10]. 